\title{
Belgeo
}

Revue belge de géographie

$1 \mid 2019$

Miscellaneous

\section{Les régions urbaines en Belgique}

Belgian urban regions

Lieve Vanderstraeten et Etienne Van Hecke

\section{(2) OpenEdition}

Journals

Édition électronique

URL : http://journals.openedition.org/belgeo/32246

DOI : $10.4000 /$ belgeo.32246

ISSN : 2294-9135

Éditeur :

National Committee of Geography of Belgium, Société Royale Belge de Géographie

\section{Référence électronique}

Lieve Vanderstraeten et Etienne Van Hecke, «Les régions urbaines en Belgique », Belgeo [En ligne], 1 | 2019, mis en ligne le 08 février 2019, consulté le 07 juillet 2020. URL : http://journals.openedition.org/ belgeo/32246 ; DOI : https://doi.org/10.4000/belgeo.32246

Ce document a été généré automatiquement le 7 juillet 2020

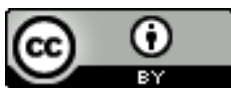

Belgeo est mis à disposition selon les termes de la licence Creative Commons Attribution 4.0 International. 


\title{
Les régions urbaines en Belgique
}

\author{
Belgian urban regions
}

\author{
Lieve Vanderstraeten et Etienne Van Hecke
}

Les auteurs remercient Mr. Youri Baeyens de la Direction Générale Statistique, Statistics Belgium, pour le calcul des agglomérations basées sur une distance de $100 \mathrm{~m}$, utilisée dans cette étude pour la définition des agglomérations des régions urbaines.

\section{Introduction}

1 Dans notre société occidentale, les grandes villes et les villes moyennes ont adopté une forme qui est non seulement beaucoup plus vaste que celle de la ville traditionnelle, mais plus vaste aussi que celle de l'habitat urbain aggloméré, l'agglomération morphologique. Ces deux concepts ne suffisent pas à cerner la ville actuelle. H. Van der Haegen a introduit pour la Belgique le concept de région urbaine dans les années 1970 (Van der Haegen, Pattyn, 1979). Van der Haegen (id.) a délimité 15 régions urbaines belges sur la base des données du recensement de 1970. La suburbanisation continue à partir des années 1960 rend la région urbaine particulièrement dynamique. Le traçage d'une frontière statique n'est pas réaliste. La délimitation d'une situation à un moment donné doit donc être régulièrement révisée. Pour être pertinente, celle-ci doit pouvoir se baser sur des données systématiquement actualisées. Ceci était jusqu'à 2001 particulièrement le cas lors des recensements. De ce fait, les régions urbaines ont été actualisées sur la base des données des recensements de 1981, 1991 et 2001 (S. Leemans et al., 1987 ; Van der Haegen et al., 1996 ; Luyten et al., 2009). Une nouvelle actualisation s'impose.

2 La première délimitation, celle de 1970, est difficilement comparable aux délimitations postérieures à cause de la fusion des communes au 1er janvier 1977. Les délimitations étaient, du fait de l'échelle des communes, plus précises à cette date. En 1981 et 1991, 17 régions urbaines furent définies. En 2001, on note une différence de composition : la ville régionale de La Louvière n'est plus retenue comme centre d'une région urbaine, sa banlieue de 1991 ayant disparu ; d'autre part, le bipôle Genk-Hasselt, retenu par H. Van 
der Haegen (Leemans et al., 1987), est scindé en deux régions urbaines, chaque ville régionale étant au centre de chacune d'elle. Cette scission était basée sur le fait que la suburbanisation de chacune des villes se dirige vers des communes distinctes et que les deux entités ainsi retenues ont une structure socio-économique différente. Finalement, la ville régionale de Turnhout est devenue en 2001 le centre d'une région urbaine et en 2017, c'est également le cas pour la ville de Roulers.

\section{Les composantes de la région urbaine}

La subdivision proposée par H. Van der Haegen et M. Pattyn (1979) était le résultat d'une approche fonctionnelle et morphologique prenant en compte la croissance de la région urbaine. Le noyau urbain est le cœur de la ville, avec la plus haute densité de commerces et services et contenant en général le centre urbain historique. Ce noyau urbain est imbriqué dans un tissu de quartiers urbains à construction très dense, correspondant en général aux extensions datant du $19^{\mathrm{e}}$ siècle. C'est une zone comptant essentiellement des bâtiments résidentiels, mais où se mêlent des activités variées, telles que commerce et artisanat, industries, écoles et hôpitaux. Dans son ensemble, la ville centrale, comprenant le noyau urbain et ces quartiers agglomérés à construction dense, peut se définir comme étant l'ancienne partie centrale agglomérée de la région urbaine. Cette ville centrale est entourée de toutes parts par la couronne urbaine constituée essentiellement de constructions du $20^{\mathrm{e}}$ siècle, toujours d'un seul tenant mais de moindre densité. La fonction principale revêt ici de toute évidence un caractère résidentiel, ce qui n'exclut pas la présence de commerces et services dans les anciens noyaux communaux et le long des axes routiers, phénomène en croissance. Dans son ensemble, l'agglomération morpholoqique, comprenant la ville centrale et la couronne urbaine, se définit comme étant un ensemble aggloméré dans l'espace. Du point de vue pratique il a paru souhaitable de faire coïncider l'agglomération et les limites des communes. Le résultat de cet ajustement est l'agglomération morphologique opérationnelle. La banlieue touche à l'agglomération: c'est la zone de croissance de la ville. La croissance y est due dans une large mesure à la suburbanisation. En dehors de la banlieue, s'étend une zone qui, à la suite d'un développement considérable du mouvement pendulaire autochtone, se rattache également à la ville. Cette zone des migrants alternants constitue avec la région urbaine le complexe résidentiel urbain.

\section{Les définitions ne sont pas universelles}

4 Le concept de région urbaine (stadsgewest, City Region, Stadtregion, S.M.S. A.Standard Metropolitan Statistical Area) se retrouve au niveau international, mais les critères utilisés pour définir la région urbaine diffèrent d'un pays à l'autre, entre autre en ce qui concerne le nombre d'habitants minimum (par exemple 1000 au Canada, 75000 en Belgique, ...). La délimitation au Canada repose sur un nombre minimum d'habitants, une densité minimale et la distance entre ces « îlots». De plus, une région urbaine peut ou non être subdivisée en zones. En Belgique la distinction est faite entre une agglomération centrale d'habitat aggloméré continu entourée d'une banlieue avec un habitat plus lâche mais dont la population, en général plus aisée, est clairement dirigée vers la ville centrale ou l'agglomération pour diverses activités. Mais aussi le fait urbain peut se retrouver sous une autre dénomination, bien que le contenu soit 
également une (grande) concentration urbaine. La combinaison d'un minimum de population et de densité est courante dans différents pays. Au Royaume-Uni, il s'agit de zones dont les intervalles entre les zones d'habitat ne dépassent pas 200 mètres. En France, on définit une "aire urbaine", qui est selon l'INSEE un ensemble continu et sans enclave formé d'un pôle urbain (« unité urbaine offrant plus de 10000 emplois ») entouré par sa "couronne périurbaine ", c'est-à-dire les communes dont $40 \%$ de la population active résidente ont un emploi dans le pôle urbain. Le concept d'«aire urbaine" en France correspond dans les grandes lignes au "complexe résidentiel urbain » retenu en Belgique et «l'unité urbaine » correspond à "l'agglomération » de la région urbaine belge. En général le terme de région urbaine recouvre une multitude de situations soutenues par un ou quelques critères combinés de nombre d'habitants, de densité, éventuellement de superficie et de distance. Dans quelques cas seulement, la délimitation repose sur un pourcentage minimum de mouvements pendulaires vers une aire centrale.

5 Le terme d'agglomération est assez semblable dans les différents pays : il s'agit d'une entité avec un habitat concentré ou/et dense ; le seuil minimum d'habitants, la distance utilisée pour définir "l'habitat continu»,... peuvent être différents. La directive européenne entend instaurer une certaine uniformité en introduisant la distance de 200 mètres comme seuil d'habitat continu. Comme nous le verrons plus loin, cette distance peut difficilement être appliquée dans tous les pays européens, en particulier dans un pays comme la Belgique avec un habitat dense mais également dispersé, ce qui mène à une agglomération d'habitat qui dépasse de loin les différentes entités urbaines géographiques (voir ci-après).

6 Le terme de banlieue par contre est moins utilisé et se rapporte à des contenus forts différents. Il a une signification géographique, celle de la ceinture urbanisée dépendant du centre, mais aussi une signification symbolique relative à la marginalité et au discrédit qui pèse sur ceux qu'on qualifie « d'exclus » par réduction hâtive (VieillardBaron, 2001). La différence entre le " contenu » de la banlieue selon les pays est liée à des processus de suburbanisation et à des aménagements du territoire différents. Avec la révolution industrielle, les grandes villes françaises par exemple, en particulier Paris, ont commencé à déverser sur leurs banlieues les populations qu'elles ne pouvaient plus loger. La banlieue y est devenue un exutoire pour les activités encombrantes ou polluantes. Par l'usage inconsidéré qui en est fait dans le cadre français, le mot banlieue renvoie non pas à une entité spatiale précise, mais à une notion vague, susceptible de s'appliquer à tout secteur enclavé et à toute population qui s'écarterait de la norme. D'autre part, dans le vocabulaire parisien, la banlieue est tout en dehors de la ville de Paris, au-delà du périphérique. Il va de soi que cette approche française de la banlieue ne peut être transposée dans le cadre européen (Vieillard-Baron, 2001). En fait, la banlieue y est une ceinture bâtie qui agglomère une très grande diversité de territoires, aussi bien des communes qui ont connu une longue histoire et sont englobées dans la croissance de l'habitat aggloméré, que de petits noyaux villageois démesurément agrandis par l'arrivée des lotissements pavillonnaires et finalement des grands ensembles datant des années 1960-70.

7 Dans les pays anglo-saxons, la notion de «suburb» recouvre des dimensions urbaines et sociales très contrastées. Elle se rapporte, le plus souvent, aux franges de la ville, et non à la ceinture fortement urbanisée qui entoure la ville-centre. En Angleterre et aux États-Unis, elle est plutôt synonyme d'opulence. Les communes de banlieue en Belgique 
sont dans la plupart des cas composées d'anciens noyaux villageois auxquels se sont ajoutés depuis les années 1960, ou plus tard pour les communes plus éloignées du centre-ville, des habitations non contiguës, soit en lotissements, soit le long de la voirie existante. Plus récemment, des immeubles à appartements se sont ajoutés dans certaines communes. Il peut y avoir une différence sociale entre la population des anciens noyaux villageois et celle des extensions récentes, plus riche. Toutefois, ces différences ont diminué au fil du temps, de nombreuses habitations anciennes ayant été rachetées et rénovées par une population jeune et plus prospère. Un habitat individuel dans un environnement vert d'une part, une population plutôt riche, sont dans le langage populaire les deux caractéristiques dominantes d'une commune de banlieue belge. Initialement, la nouvelle population était jeune, mais maintenant elle a vieilli dans les communes les plus anciennement suburbanisées.

8 La définition d'Eurostat correspond grosso modo au complexe résidentiel urbain de Belgique en ce qui concerne la délimitation extérieure (une zone de navetteurs avec communes contiguës avec $20 \%$ de la population active travaillant dans la ville centrale). Les subdivisions à l'intérieur de la "Functional urban area", auparavant «Larger Urban Zone » sont différentes de notre subdivision. Il nous semble plus logique de considérer les navettes vers l'agglomération que seulement vers la ville centrale, vu l'évolution des localisations des activités économiques. Tandis que le concept d' agglomération (habitat séparé par moins $200 \mathrm{~m}$ ) apparaît comme une définition chez Eurostat, le terme de banlieue n'y apparaît pas.

\section{La méthode de délimitation des régions urbaines belge 2017}

9 La délimitation 2017 (appelée ainsi sur la base de la date de la plupart des données utilisées) effectuée en 2018 utilise les données disponibles les plus récentes, mais cellesci ne correspondent pas toujours à la même date.

\section{La ville centrale}

10 La description des composantes de la région urbaine citée plus haut est basée sur une situation de développement urbain datant d'il y a plus de quarante ans. La délimitation extérieure de la ville centrale se faisait $(1970,1981,1991)$ sur la base de différents critères: densité de la population d'une part, d'autre part caractéristiques des logements (peu de maisons unifamiliales, un pourcentage élevé d'habitations construites avant 1945 et un pourcentage important d'habitations avec une surface inférieure à $45 \mathrm{~m}^{2}$ ). Depuis, les centres urbains ont subi des changements importants, en particulier de rénovation urbaine en ce qui concerne l'habitat. De plus, certains indices n'étaient déjà en 2001 plus disponibles ou fiables, en particulier la superficie et l'âge des habitations. Pour toutes ces raisons, les centres-villes n'ont plus été définis depuis 1991. Pour 2017 nous nous limiterons donc à la délimitation de l'agglomération morphologique (opérationnalisée) et de la banlieue, mais également avec une distinction à l'intérieur de l'agglomération morphologique entre la ville centrale administrative (commune) et la couronne d'habitat continu ramenée au niveau communal, qui forme le reste de l'agglomération. Pour les villes moyennes, la ville administrative et l'agglomération morphologique opérationnalisée se juxtaposent. 


\section{La délimitation des agglomérations}

11 La limite extérieure de l'agglomération morphologique ou du caractère continu de l'habitat est traditionnellement le résultat d'un relevé sur le terrain ou d'analyse de photographies aériennes. Lors des recensements de 1991 et de 2001, l'habitat continu fut relevé pour toutes les entités d'habitat aggloméré et fut publié sous de le nom de "noyau d'habitat» (Mérenne-Schoumacker et al., 1998). Vu le manque de moyens financiers, il n'était pas possible d'utiliser une délimitation au départ de photographies aériennes ou d'images satellitaires, une procédure bien trop longue. Une autre source moins intensive en travail devait donc être recherchée. Une opportunité s'offrait: la délimitation des "agglomérations" dans le cadre du règlement communautaire européen CE N ${ }^{\circ}$ 1201/2009 fixant les spécifications techniques du recensement de la population et des logements. C'est aussi la définition recommandée depuis 1978 par les Nations-Unies. L' "agglomération », appelée aussi «localité " y est définie comme un ensemble de constructions dont aucune n'est distante de plus de 200 mètres de la construction la plus proche. La notion d'agglomération ne dépend donc pas des limites administratives : une "agglomération ou localité » peut donc s'étendre sur plusieurs communes et une commune peut contenir plusieurs localités. Celles-ci ont été déterminées par la Direction générale Statistique, Statistics Belgium (Statistics Belgium, open data). À cet effet un inventaire de tous les couples de logements distants de moins de $200 \mathrm{~m}$ a été créé. Un algorithme a alors été appliqué afin de déterminer le contenu des différentes localités (Statistics Belgium, 2016). Le nombre total d'agglomérations s'élève à $20768: 19262$ de moins de 200 habitants, une de plus d'un million d'habitants comptant pas moins de 5901474 individus ou plus de la moitié de la population de la Belgique. Cette méga-agglomération couvre une grande majorité de la Flandre, de Bruxelles-Capitale et aussi une partie de la Wallonie. 280 communes sont concernées partiellement ou totalement par cette agglomération. Ceci n'exclut pas la présence d'autres localités dans certaines de ces communes: ce sont des îlots à l'intérieur de la mega-agglomération formée par des concentrations d'habitations dont chaque habitation est éloignée de plus de 200 mètres d'une quelconque habitation de la méga-agglomération.

12 L'existence d'une méga-agglomération reflète la structure de l'habitat en Belgique et particulièrement en Flandre. Celle-ci est due d'une part à la structure de l'habitat rural ancien dans certaines régions de Flandre et à l'absence pendant longtemps de règlements d'aménagement du territoire. De ce fait, des habitations ont été construites entre l'habitat rural ancien, tandis que l'habitat en ruban s'est fortement développé au départ des villes et villages. Géographiquement, sociologiquement et du point de vue de l'aménagement du territoire et politique de la ville, une telle délimitation n'est ni réaliste, ni souhaitable. De toute façon, elle ne peut être utilisée dans le cadre de la définition d'agglomérations des régions urbaines.

13 Afin de mieux cerner la réalité de la structure belge, Statistics Belgium a calculé, dans le cadre de notre délimitation des régions urbaines, selon le même algorithme, les agglomérations sur la base d'une distance de 100 mètres par rapport à la construction la plus proche $^{1}$. Le revers de cette plus petite distance est la scission d'une agglomération géographique en deux ou plusieurs agglomérations statistiques par la présence d'une autoroute, d'un canal ou de bâtiments industriels d'une largeur de plus de 100 mètres. En effet la délimitation d'une agglomération est basée sur les 
coordonnées des habitations. D'autres éléments construits ne sont pas pris en compte. Il en va de même pour les terrains sportifs, un parc, etc. Ceci est pris en compte plus loin dans la méthode de travail. La délimitation des localités se faisant au niveau des habitations, la délimitation des localités ne coïncide pas avec des limites statistiques ou administratives. Afin d'être opérationnelle, la délimitation a été rapportée ici au niveau des secteurs statistiques. Le secteur statistique est rattaché à une localité limitrophe sur la base de la plus grande part de la population du secteur. Des essais à $125 \mathrm{~m}$ et 150 $\mathrm{m}$ ont permis de choisir la distance de $100 \mathrm{~m}$ pour éviter l'effet de l'urbanisation en ruban. Il est plus aisé et opportun, par la suite, d'agglomérer éventuellement plusieurs localités s'il apparaît que les scissions peuvent être rattachées à un phénomène urbain.

\section{La délimitation des agglomérations en pratique : méthodologie}

14 Vu la méthode détaillée utilisée en 2001, on est parti ici du postulat que l'agglomération actuelle correspond au minimum à cette délimitation. Ceci permet de considérer que la ou les localités de Statistics Belgium de 2017 recouvrant le noyau d'habitat 2001 fait ou font partie de la nouvelle agglomération 2017. Il ne faut pas à ce niveau se soucier de ruptures internes à cause de surfaces urbanisées non habitées.

Dans la plupart des cas, les localités 2017 de Statistics Belgium (SB) ne sont pas identiques au noyau d'habitat défini en 2001. Plusieurs situations peuvent être relevées :

- Dans le cas où une localité SB 2017 dépasse le noyau d'habitat 2001, ces secteurs statistiques qui viennent s'ajouter sont pris en compte à condition que la densité de population y dépasse les 500 habitants par $\mathrm{km}^{2}$.

- Dans les cas où une autre localité SB est attenante au noyau déjà défini, ce qui veut dire qu'il existe un espace non habité d'un minimum de 100 mètres, l'affectation du sol dans cette «rupture » est examinée sur des photographies aériennes. Si cet espace est urbanisé, les secteurs statistiques contigus avec une densité de population de $>500$ habitants $/ \mathrm{km}^{2}$ sont rattachés à l'agglomération morphologique. Ceci signifie que la partie de la localité SB avec une densité moins dense ou les secteurs denses mais isolés par rapport à la partie déjà rattachée n'est pas reprise dans l'agglomération morphologique. Donc, la contiguité et la densité de population sont imposées pour la prise en compte des différents secteurs statistiques. Le seuil de densité a été introduit pour réduire les effets néfastes de l'habitat diffus sur le processus de délimitation. Notons que la densité moyenne des communes d'agglomération (hors de la ville centrale) s'élève à 890 hab. $/ \mathrm{km}^{2}$ mais les franges, aussi celles définies auparavant, sont toujours moins denses. La densité de population de la banlieue s'élève en moyenne à 297 habitants par $\mathrm{km}^{2}$ mais est plus élevée dans sa partie bâtie, d'où le choix de 500 habitants $/ \mathrm{km}^{2}$.

16 Finalement, l'opérationnalisation de l'agglomération correspond à un ajustement aux limites communales : au minimum, $50 \%$ de la population communale doit habiter dans le noyau d'habitat déterminé suite à la méthode décrite. La priorité a été donnée ici à la méthode suivie lors les précédentes délimitations, mais adaptée aux nouvelles données disponibles chez Statbel. D'autres techniques (méthode fractale) permettent une délimitation non dépendante de seuils de valeurs définis a priori (Thomas, 2012). 


\section{La délimitation de la banlieue}

17 Tenant compte de l'évolution sociétale d'une part, de la pertinence de certains indicateurs de l'autre, la composition des indicateurs a été légèrement modifiée par rapport à 2001. La définition de la banlieue se faisait jusque 2001 sur la base de la croissance de la population, d'un revenu médian plus élevé que celui de l'arrondissement, des migrations venant de l'agglomération ou partant vers la commune centrale, des navettes de travail et scolaires, de la part de la surface bâtie affectée à l'habitat ou de son évolution suffisamment forte. Pour la superficie bâtie, nous nous limitons à la dynamique, sans tenir compte de l'état des choses, parfois moins significatif (à cause de la présence de bois, etc.). À cause de l'immigration étrangère dans certaines agglomérations, le revenu y a baissé et également celui de l'arrondissement: de ce fait de nombreuses communes (également dans la zone des mouvements pendulaires) ont un revenu supérieur à la moyenne de l'arrondissement. De ce fait, il a été décidé d'attribuer un poids plus important aux flux de relations entre communes et agglomération. Les flux migratoires ont été dédoublés en ce sens que le choix est ouvert entre la migration totale et/ou un flux migratoire spécifique lié à l'âge, 20 à 24 ans vers la ville centrale (2 $2^{\mathrm{e}}$ génération), 30-39 ans de l'agglomération vers les communes extérieures (suburbanisation). Le groupe d'âge des 25-29 ans n'est pas pris en compte car il peut y avoir un mélange des flux dans les deux sens. Les critères utilisés sont les suivants :

- L'accroissement du nombre de ménages entre 2001 et 2016 est égal ou supérieur à 115 (indice d'accroissement = ménages 2016/ménages $2001 \times 100)$ (1,5 points) ;

- L'accroissement de la surface bâtie dans la commune entre 2001 et 2015 (surface bâtie 2015/ surface bâtie $2001 \times 100$ ) est égal ou supérieur à 116,5\% pour les communes wallonnes, et à $114,2 \%$ pour les communes flamandes. Ces pourcentages correspondent aux évolutions respectives dans les deux régions (1,5 points) ;

- La migration moyenne provenant de l'agglomération est, pour la période 2006-2016, égale ou supérieure à $40 \%$ de l'immigration dans la commune pour le total ou pour le groupe des 30 à 39 ans (2 points) ;

- L'émigration moyenne vers la ville centrale (ou dans le cas de Bruxelles, l'ensemble de la Région de Bruxelles-Capitale) au départ de la commune est, pour la période 2006 -2016, égale ou supérieure à $25 \%$ de la migration totale ou de celle dans le groupe des 20 à 24 ans (2 points);

- La navette de travail vers l'agglomération est, en 2014, égale ou supérieure à $30 \%$ de la population active occupée habitant la commune ou la navette vers l'agglomération est, en 2014, égale ou supérieure à $50 \%$ des migrants alternants sortants (population active habitant la commune et travaillant hors de la commune) (données ONSS) (3 points) ;

- La navette scolaire des étudiants du secondaire vers l'agglomération est, en 2015-16, égale ou supérieure à $35 \%$ de la population scolaire totale (du niveau secondaire) habitant la commune (3 points).

18 Le maximum de points est de 13 ; les communes dépassant la moitié de ce score sont considérées automatiquement comme commune de banlieue. Mais pour les communes appartenant déjà à la banlieue le critère est moins sévère : la commune reste dans la banlieue quel que soit le score total, mais à la condition que deux flux atteignent les seuils; donc une combinaison d'au moins un flux de migration (critère 3 et/ou 4) avec 
un flux de travail (critère 5) ou scolaire (critère 6), ou si le seuil est dépassé pour un flux de travail et le flux scolaire. En pratique cela ne concerne que 7 communes.

\section{La délimitation de la zone des migrants alternants}

$19 \mathrm{Vu}$ la disponibilité des données, les mouvements pendulaires sont calculés sur la base des travailleurs salariés repris dans les statistiques de l'ONSS, tandis qu'auparavant la délimitation était basée sur les chiffres de la population active des recensements. Les indépendants ne sont pas repris dans ces chiffres de l'ONSS, le seuil de $15 \%$ (utilisé précédemment) a été relevé à $20 \%$.

\section{Les régions urbaines 2017}

\section{L'évolution générale}

19 villes sont au centre d'une région urbaine (tableau 1 et figure 1). Ces 19 communes incluses, les agglomérations des régions urbaines comptent 115 communes entourées de 99 communes de banlieue, soit 214 communes pour les régions urbaines. La zone résidentielle des migrants alternants compte 162 communes, soit un total de 376 communes pour les complexes résidentiels urbains. $36 \%$ des communes belges font donc partie des 19 régions urbaines, auxquelles s'ajoutent près d'un quart des communes dont la population est orientée dans une certaine mesure pour les flux de travail vers ces 19 agglomérations urbaines.

21 L'évolution est intéressante, bien qu'elle puisse être légèrement influencée par certaines modifications de la méthodologie. Le nombre de communes des régions urbaines s'élevait à 218 en 2001. 16 communes se sont ajoutées aux agglomérations; à part la ville de Roulers, il s'agit de 15 communes de banlieue qui ont été morphologiquement absorbées entre ces dates. D'autre part, la banlieue compte moins de communes (99 au lieu de 119) : le nombre de communes passant à l'agglomération (15) est pratiquement compensé par celui des communes venant de la zone des migrants alternants (16), auxquelles s'ajoutent 4 nouvelles communes (dont 2 de Roulers); mais on note 25 communes faisant partie de la banlieue en 2001 qui ne remplissent plus les conditions pour être considérées comme communes de banlieue, et ce malgré un abaissement important du score requis pour les communes faisant déjà partie de la banlieue. Il est frappant que 15 de ces 25 communes sont situées dans la banlieue de Bruxelles et de Liège. Ces 25 communes n'ont plus que des flux relationnels pour le travail, les flux en relation avec l'enseignement secondaire ou migratoires (dans les deux sens) ne ressortant plus. Ainsi, les complexes résidentiels urbains sont passés de 366 à 376 communes.

\section{La composition des agglomérations 2017}

Les agglomérations des régions urbaines de Wallonie sont en 2017 identiques à celles de 2001. Il se peut que l'agglomération morphologique dépasse dans quelques cas les limites de l'agglomération opérationnelle, mais la partie située en dehors de ces limites compte moins de $50 \%$ de la population de la commune (Fleurus, Aiseau-Presles, Hamsur-Heure-Nalinnes pour Charleroi ; Juprelle pour Liège ; Herve pour Verviers). 
23 En Flandre par contre, on note l'extension de plusieurs agglomérations. Les agglomérations de Bruges, Genk, Louvain, Ostende, Saint-Nicolas et Turnhout restent identiques. Une petite partie de Tamise se rattache à l'agglomération morphologique de Saint-Nicolas, mais nettement en dessous du seuil de $50 \%$ de la population communale.

24 L'habitat aggloméré de Wavre-Saint-Catherine est en continuité avec le noyau d'habitat de Malines et concentre plus de $50 \%$ de la population communale. La commune est donc rattachée à l'agglomération de Malines. Ce n'est pas le cas pour Zemst, la partie accolée à Malines étant en dessous du seuil de 50 \%. Pour les mêmes raisons, Zonhoven est repris dans l'agglomération de Hasselt, tandis que ce n'est pas le cas pour Diepenbeek. Wevelgem est repris dans l'agglomération de Courtrai, à l'inverse de Zwevegem ( $<50 \%)$. Wevelgem forme pour Statistics Belgium une localité séparée, mais elle est géographiquement rattachée à l'agglomération de Courtrai par des infrastructures routières et industrielles.

Les agglomérations des deux grandes villes de Flandre s'agrandissent de plusieurs communes. Pour la ville de Gand c'est le cas avec l'absorption d'Evergem, de Lochristi et de Melle. Il s'agit chaque fois d'une agglomération séparée pour Statistics Belgium, mais la séparation est due à l'absence d'habitat dans des zones urbanisées (infrastructures routières et fluviales, zones industrielles). L'extension de la " localité (SB) » sur la commune de Destelbergen concerne moins de $50 \%$ de la population de cette commune. Pour la ville d'Anvers, 6 communes viennent s'ajouter à l'agglomération: il s'agit de Wijnegem, Kontich et Zwijndrecht, dont l'habitat aggloméré est séparé de l'agglomération actuelle par une urbanisation inhabitée (infrastructures routières et industrie). Schilde est en continuité morphologique avec Wijnegem et Lint avec Kontich, d'où le rattachement de ces deux communes. La partie de Stabroek rattachée à l'agglomération morphologique d'Anvers a grandi et concentre plus de $50 \%$ de sa population, d'où le rattachement de cette commune.

26 L'agglomération de la région urbaine de Bruxelles s'agrandit également. Par la croissance ou la densification de l'habitat, les communes d'Asse et de Kortenberg sont rattachées à l'agglomération. En ce qui concerne Steenokkerzeel, il s'agit du rattachement de ce que SB considère comme une autre agglomération, mais l'habitat de cette concentration est rattaché à l'agglomération de Bruxelles par le fait que cette coupure provient de la présence de l'aéroport de Zaventem.

\section{Les agglomérations 2017 : un tissu morphologique et socio- économique hétérogène.}

La croissance de l'habitat sur le territoire de certaines anciennes communes de banlieue a mené, au fil du temps, à l'agrandissement de la superficie de «l'habitat continu ", c'est-à-dire de l'agglomération morphologique. Comme suggéré dans la phrase précédente, des communes caractérisées comme étant de banlieue lors des délimitations précédentes font partie en 2017 de l'agglomération et en faisaient déjà partiellement partie en 2001 ou avant. La morphologie primant dans la délimitation de l'agglomération, celle-ci réunit des communes de caractéristiques différentes. Ce phénomène fut déjà étudié pour l'agrandissement de l'agglomération entre 1981 et 2001. Les communes qui en 1981 faisaient partie de la banlieue et qui en 2001 étaient absorbées par l'agglomération présentaient des caractéristiques démographiques et socio-économiques diverses (Van Hecke, Vanderstraeten, 2016). La plupart des 
communes s'ajoutant à l'agglomération ont par exemple une densité de population moins élevée : les communes qui se sont ajoutées entre 1981 et 2001 avaient en 2011 une densité de population de 814 habitants par $\mathrm{km}^{2}$, contre 996 pour les communes d'agglomération de 1981. Toutefois, la règle de moindre densité n'est pas absolue : il est possible que, par le biais de la croissance morphologique, une commune avec des caractéristiques autres que celles qui caractérisent en moyenne la commune de banlieue, soit rattachée à l'agglomération. Si en moyenne, la population des communes rattachées à l'agglomération entre 1981 et 2001 a des similitudes avec la moyenne de la banlieue, elle est toutefois moins jeune que la population des communes de banlieue de 2001. Morphologiquement, ces communes récemment englobées sont également différentes : une partie de l'habitat est plus récent, avec plus de villas, d'où un habitat moins dense.

Les communes rattachées à l'agglomération de 2017 présentent également des caractéristiques différentes. Certaines (qui étaient des communes de banlieue en 2001) étaient plutôt rurales, mais la partie touchant à l'agglomération s'est fortement urbanisée, d'où le rattachement morphologique : c'est par exemple le cas pour WavreSaint-Catherine (Malines) et Lochristi (Gand). D'autres avaient déjà connu l'implantation de grands lotissements, avec une population typiquement de «banlieue»; c'est par exemple le cas de Steenokkerzeel (Bruxelles), Kontich et particulièrement Schilde (Anvers). D'autres communes encore, englobées par la croissance de l'habitat, peuvent correspondre à un ancien noyau, avec une population importante et un habitat dense, comme c'est le cas pour la commune d'Asse, petite ville à l'ouest de Bruxelles.

\section{L'agglomération élargie de Bruxelles}

La délimitation de l'agglomération de Bruxelles vers le sud-est exige une approche méthodologique propre. L'agglomération présente une forme asymétrique à cause de la présence de la forêt de Soignes, qui empêche une croissance concentrique uniforme. Vandermotten et al. (1999) considèrent que les communes de La Hulpe et Rixensart en Brabant wallon et de Hoeilaart et Overijse en Brabant flamand font partie de l'agglomération morphologique. Thomas et al. (2011) considèrent dans leurs représentations cartographiques du volume Habitat de l'Atlas de Belgique que les communes de La Hulpe, Rixensart et Lasne d'une part, d'Overijse et de Hoeilaart de l'autre, font partie de l'agglomération bruxelloise. Ces auteurs suivent le principe introduit par H. Van der Haegen (1995) de "l'agglomération bruxelloise étendue " basée en premier lieu sur la morphologie mais tenant compte du fait que ces communes situées de l'autre côté de la forêt de Soignes présentent dans les grandes lignes les mêmes caractéristiques que les communes situées à une distance quasi égale de Bruxelles dans les autres directions. La forêt de Soignes n'est dans ce cas pas un obstacle qui définit la limite de l'agglomération mais est à considérer comme un vaste parc urbain.

Dans l'esprit de la méthodologie suivie ici, il n'y a pas de rattachement de l'habitat d'Overijse par l'intermédiaire de Tervuren. Au sud, une partie de Lasne relie Waterloo à (Genval -)Rixensart et de là à Wavre, à l'intérieur d'une "agglomération Statbel». Toutefois la population de Lasne concernée est en dessous des $50 \%$ de la population communale. De ce fait, il y a une rupture de l'agglomération morphologique entre 
Waterloo et Rixensart. En fait, cette rupture est liée au fait que les secteurs statistiques du nord-ouest de Lasne n'atteignent pas une densité de plus de $500 \mathrm{hab} . / \mathrm{km}^{2}$, seuil requis dans notre méthodologie pour être considéré comme "agglomération ». Il y a donc une rupture dans la continuité de l'habitat aussi bien à l'est qu'au sud. Une partie de l'habitat des communes de Hoeilaart, Overijse et la Hulpe est jointif à la forêt de Soignes mais dans la méthodologie générale, seules des ruptures de caractère urbain ont été prises en compte.

On peut définir, séparément, une "agglomération élargie ", formée des communes d'Overijse, Hoeilaert, La Hulpe, Lasne, Rixensart et Wavre, mais il n'est pas conséquent, avec la méthodologie adoptée ici, de considérer cet ensemble comme une extension de l'agglomération bruxelloise. Celle-ci est également reprise au tableau 1, mais non insérée dans les tableaux chiffrés.

\section{La composition de la banlieue 2017}

Certaines communes de banlieue de 2001 sont devenues des communes d'agglomération, d'autres se sont ajoutées, d'autres encore ne peuvent plus être considérées comme commune de banlieue parce qu'elles ne répondent plus aux critères définis, malgré un score moins exigeant pour les communes de banlieue déjà retenues en 2001. Ce phénomène est spatialement concentré, puisque parmi ces 25 communes, huit (dont 7 dans le Brabant wallon) appartenaient à la région urbaine de Bruxelles, 7 à celle de Liège et 3 à celle de Charleroi. En ce qui concerne la banlieue bruxelloise, 5 communes (Ottignies-Louvain-La-Neuve, Wavre, Chaumont-Gistoux, Beauvechain, Grez-Doiceau) sont contiguës et forment une entité qui s'oriente vers les services et commerces de Wavre et Louvain-la-Neuve. Pour la région urbaine de Liège, la dispersion des communes est plus grande. Il s'agit d'une série de communes périphériques. Pour Charleroi, il s'agit d'une dynamique négative de la région urbaine.

Les nouvelles communes de banlieue sont par contre réparties sur un grand nombre de régions urbaines. La croissance la plus forte de la banlieue caractérise les régions urbaines de Bruges et Tournai, avec 3 nouvelles communes. Pour les grandes villes, aucune nouvelle commune de banlieue ne s'ajoute à Anvers, Liège et Charleroi ; on en compte une de plus pour Bruxelles et 2 pour Gand.

\section{La composition de la zone des migrants alternants 2017}

Le nombre de communes appartenant à une zone des migrants alternants résulte de quatre mouvements:d'une part des communes dont le seuil des mouvements pendulaires vers l'agglomération est atteint, il s'agit éventuellement d'une « nouvelle » commune de résidents alternants ou, à l'inverse, le flux est devenu relativement moins important vers l'agglomération et la commune est rayée de la composition. D'autre part, par rapport à la banlieue : certaines communes de la zone en 2001 atteignent en 2017 certaines caractéristiques de commune de banlieue et sont reprises dans cette zone. Enfin, les conditions pour appartenir à la banlieue ne sont plus remplies par certaines communes, qui sortent de la banlieue et deviennent communes de la zone des migrants alternants, la seule caractéristique requise étant alors le pourcentage de navetteurs de travail. 
L'étendue et la dynamique de la zone des migrants alternants est le reflet de l'importance de l'emploi dans les agglomérations. Il n'est pas étonnant que la moitié des communes de cette zone se retrouve dans la zone d'attraction de Bruxelles et que les villes régionales n'ont qu'une zone d'attraction de navetteurs peu étendue. Parmi celles-ci, Namur connaît la plus grande progression. Les crises de l'emploi qui ont affecté Genk ou Charleroi illustrent ce phénomène : moins de communes composent la zone de Genk et, en ce qui concerne Charleroi, une commune de banlieue en 2001 (Les Bons Villers) et une autre qui appartenait à la zone des navetteurs (Pont-à-Celles), toutes deux situées au nord de Charleroi, font maintenant partie de la zone des migrants alternants de Bruxelles. Il en va de même pour Fosses-la-Ville, qui passe dans la zone d'attraction de Namur et finalement Sambreville disparait de la catégorie.

\section{La comparaison 2001-2017, analyse statistique}

même proportion qu'en 2001, sur une superficie légèrement plus petite : $26 \%$ au lieu de $27 \%$ de la superficie du pays. Il y a donc, tenant compte de l'évolution de la population totale du pays, une densification: 799 habitants par $\mathrm{km}^{2}$ en 2017 par rapport à 709 en 2003. La population des villes centrales a augmenté de $10 \%$ (en ne tenant pas compte de Roulers), celle des agglomérations entières de $16 \%$, mais ici intervient une augmentation de la superficie de $11 \%$ due à l'insertion d'anciennes communes de banlieue. La superficie de la banlieue est de $18 \%$ plus petite en 2017 par rapport à 2001, la population de $15,5 \%$. La densité de population passe donc de 290 à 297 habitants par km² ${ }^{2}$ toutefois avec une composition partiellement différente. La zone des migrants alternants connaît une augmentation de densité de 275 hab. $/ \mathrm{km}^{2}$ en 2001 à 280 en 2017 et ce avec une population de 6,4\% supérieure sur une superficie de 4,7\% plus grande. La densification se fait donc dans les agglomérations et plus spécifiquement dans les villes centrales.

38 
régresse et ces communes passent dans la zone des migrants alternants qui s'agrandit. De ce fait la superficie des régions urbaines de Bruxelles et de Wallonie régresse, mais celle des complexes résidentiels urbains se maintient.

La densité de population montre deux gradations (tableau 5) : l'une du centre vers la périphérie et l'autre en fonction de la hiérarchie urbaine, avec une exception toutefois : la zone des migrants alternants d'Anvers a une densité plus forte que la banlieue de cette ville, et de là également pour les moyennes de Flandre. La forte densité de la Région de Bruxelles-capitale par rapport aux autres grandes villes est en partie causée par la présence d'installations portuaires et d'industries dans ces grandes villes, à la différence de Bruxelles.

Les régions urbaines concentrent $64 \%$ de l'emploi et la zone des migrants alternants encore $15 \%$ supplémentaires (tableau 6). Les 19 villes-centres considérées (Région de Bruxelles-capitale pour Bruxelles) concentrent à elles-seules $44 \%$ de l'emploi pour $31 \%$ de la population de la Belgique. Elles attirent donc un grand nombre de navetteurs. Pour l'ensemble des autres communes des agglomérations, on est plus ou moins à l'équilibre entre la population active de résidence et celle de travail, mais dans les autres zones, la part de la population active de résidence excède la part de l'emploi salarié (banlieue, zones des migrants alternants et l'ensemble du reste du pays). La population active de ces zones est orientée vers un emploi aussi bien dans la zone ellemême que dans les villes centrales, mais aussi, dans un mesure moindre, dans les autres zones, les mouvements pendulaires étant présents partout sur le territoire. La forte dispersion en Belgique de la population et de l'emploi explique ces chiffres. Pour obtenir une délimitation des zones complexes des mouvements pendulaires, une autre approche est nécessaire: non une orientation vers une agglomération centrale mais une analyse de réseaux (Verhetsel et al., 2018).

41 Le tableau 6 montre également un rapport de l'emploi nettement plus faible par rapport à la population en Wallonie que dans le reste du pays. La répartition relative de l'emploi dans les différentes zones (agglomérations, régions urbaines etc.) est quasiment similaire à celle de 2001. Les différences se situent au niveau régional, avec des évolutions négatives ou faibles dans plusieurs agglomérations de Wallonie; Namur fait exception. Dans les agglomérations de Bruxelles et des villes flamandes, l'évolution est nettement positive.

Le revenu par habitant montre des différences importantes (tableau 7), résultant de facteurs complexes. En premier lieu, on note les différences entre régions urbaines. Celles-ci sont en grande partie le reflet des activités économiques et leur évolution. Ainsi, on note des revenus peu élevés dans les anciennes agglomérations industrielles de Wallonie et les revenus y diffèrent peu entre la ville centrale et l'agglomération. L'histoire différente de Namur et sa fonction actuelle de capitale régionale tranchent avec les autres villes ou agglomérations. Par contre, en Flandre et dans la région urbaine de Bruxelles, les revenus sont nettement plus élevés dans les communes d'agglomération entourant la ville. Le mécanisme est double : certaines villes ont connu une forte immigration étrangère pesant sur la moyenne des revenus tandis que l'accroissement de l'agglomération a englobé des communes riches, au départ des communes de banlieue. Ceci explique que les revenus moyens sont dans les banlieues de Wallonie nettement plus élevés que dans les agglomérations, tandis qu'en Flandre la moyenne est même plus basse pour la banlieue que pour les communes d'agglomération hors la ville centrale. En 2015, les revenus moyens par habitant de la 
banlieue étaient de $25 \%$ plus élevés que ceux de la ville centrale, contre $14 \%$ en 2001, traduisant l'appauvrissement des villes centrales. Soulignons finalement que les revenus moyens des zones de migrants alternants ne sont que faiblement plus bas ( $3 \%$ en $2015,7 \%$ en 2001) que ceux de la banlieue. Hormis les villes centrales, on assiste donc à un nivellement des revenus dans les différentes parties des complexes urbains.

L'évolution démographique des agglomérations a entrainé un appauvrissement de celles-ci, en particulier dans leur centre. Par rapport à la moyenne du revenu moyen de la Belgique, la moyenne des agglomérations de 1991 s'élevait à $103 \%$, à $100 \%$ en 2001 et à $94,5 \%$ en 2015. Pour les villes centrales, ces pourcentages s'élèvent respectivement à $101 \%, 97 \%, 89 \%$. Il est donc clair que les bouleversements se sont situés dans les villes centrales. En 1991, le revenu moyen y était encore supérieur à la moyenne nationale ! D'autre part, la banlieue a peu changé par rapport à la moyenne nationale ; on y note cependant une légère augmentation (1991: $107 \%$ du revenu moyen national ; 2001 : $110 \% ; 2015$ : $111 \%)$.

À l'intérieur de l'agglomération, hormis la grande différence qui s'est creusée entre la ville et le reste de l'agglomération, il n'y a pas de systématique dans les différences de revenu entre les différentes parties de ces communes d'agglomérations, qu'elles soient de 1970, ajoutées entre 1970 et 2001 ou après 2001. Les différences de revenus diffèrent selon les régions urbaines.

\section{Dynamique démographique 1970-2017 à l'intérieur des agglomérations et des régions urbaines}

\section{La dynamique des agglomérations}

Pour mieux cerner la dynamique des régions urbaines, une évolution sur une période plus longue s'impose. Pour ce faire, les agglomérations de 1970 ont été transposées au niveau des communes après fusion. À cette date l'agglomération a été délimitée sur la base des anciennes communes. Si la population des anciennes communes dépasse à cette date les $50 \%$ de la population de la nouvelle commune de 1977, cette nouvelle entité a été considérée comme faisant partie de l'agglomération : ceci permet de faire une comparaison entre les communes de l'agglomération de 1970 et celles de 2017. D'autre part, toutes les communes de 2017 ne faisant pas encore partie de l'agglomération de 1970 peuvent être considérées dans leur ensemble comme l'extension de l'agglomération après cette date. Une difficulté pour la comparaison des deux entités sur le plan démographique est causée par la forte immigration à partir de 2000 , surtout dans certaines villes centrales de Flandre et certaines communes de Bruxelles.

Le tableau 8 reprend l'évolution de la population à l'intérieur des agglomérations comme définies pour 2017, et ce avec une subdivision spatiale comprenant la ville centrale (comme définie après la fusion des communes), l'agglomération de 1970 et les autres communes de l'agglomération qui se sont ajoutées ensuite. Se limiter pour l'étude de la dynamique aux seules dates de 1970 et 2017 donnerait une vue tout à fait erronée de l'évolution. En effet, la période se scinde en deux : la première période est celle de l'exode depuis les centres-villes, la deuxième celle d'un accroissement de ceuxci. Pour ne pas surcharger l'analyse, la population du recensement de 2001 a été choisie comme date-charnière. Pour la plupart des villes, c'est à cette date de recensement 
qu'un minimum de population est atteint (pour une minorité ce minimum se situe en 1991). L'analyse est complexe : les évolutions sont différentes selon les villes. Il y a une différence entre les deux grandes villes de Flandre et Bruxelles d'une part, les grandes villes de Wallonie d'autre part et finalement les villes régionales. L'évolution des villes de Wallonie est clairement influencée par leur situation économique : non seulement les villes centrales, mais également les autres communes de l'agglomération de 1970 voient leur population diminuer après 1970, contrairement à ce qui se passe en Flandre et Bruxelles, où la diminution de population se limite à la ville centrale. Le $21^{\mathrm{e}}$ siècle amène un tournant dans l'évolution: la population des villes augmente et ce proportionnellement plus en Flandre et à Bruxelles qu'en Wallonie et ce à cause d'une augmentation prononcée de la population étrangère. Toutefois on note également un accroissement de la population belge dans les villes et dans leurs agglomérations, une influence des naturalisations étant toutefois à prendre en compte. L'accroissement total après 2001 est relativement plus prononcé dans les villes que dans le reste de l'ancienne agglomération de 1970 et même que dans le reste de l'agglomération plus récente. Finalement on assiste dans les villes à un accroissement de la population plus important que celui des ménages tandis que l'inverse est observé dans le reste de l'agglomération et dans la banlieue.

\section{La dynamique des régions urbaines}

47 L'évolution de la population dans les régions urbaines et leurs subdivisions est au $21^{\mathrm{e}}$ siècle toute autre qu'au $20^{\mathrm{e}}$. Les tableaux 9 et 10 sont basés sur les compositions des régions urbaines 2017, avec les chiffres de population aux différentes dates. La première période (1970-2001) correspond pour les grandes villes à la dépopulation des villes centrales tandis que le reste de l'agglomération continue de croître, et c'est encore plus le cas pour la banlieue. Toutefois, la population totale de la région urbaine n'a pratiquement pas augmenté en 30 ans. Les régions urbaines des villes régionales de Flandre connaissent une évolution plus positive. Les problèmes économiques se reflètent dans les chiffres de la Wallonie, tant dans les villes que dans leur agglomération. La moyenne des villes régionales est positivement influencée par l'évolution de la ville de Namur, qui fait exception en Wallonie. La zone des migrants alternants connaît pratiquement partout une évolution nettement positive, moins forte que celle de la banlieue, sauf en Wallonie.

L'évolution 2001-2017 est tout différente. Les villes centrales connaissent maintenant un net accroissement, particulièrement à Bruxelles et dans les deux grandes villes de Flandre. Exprimé en évolution annuelle, la croissance du reste des agglomérations et des banlieues à Bruxelles et en Flandre est moins importante qu'avant 2000. Les agglomérations de Wallonie connaissent maintenant un accroissement, aussi bien dans les villes centrales que dans le reste de l'agglomération. Une autre différence importante est un accroissement plus important dans la zone des migrants alternants après 2000 et même des valeurs de croissance du même ordre de grandeur et parfois même plus forte que dans la banlieue, alors qu'elle était nettement moins forte par rapport à la banlieue avant 2000. Cette tendance n'est évidemment pas favorable, puisqu'elle signifie des déplacements plus longs vers l'agglomération. 


\section{Les régions urbaines, des entités pertinentes?}

Il ressort des délimitations que la plupart des villes régionales sont caractérisées par une urbanisation qui dépasse les limites administratives de la commune centrale : les entités morphologiques ou agglomérations forment ainsi la partie centrale des régions urbaines. La délimitation d'une urbanisation continue nous semble importante en ce sens que le critère de l'habitat continu est internationalement utilisé, en particulier par Eurostat, selon différentes variantes. En Belgique, la densité et surtout l'implantation de l'habitat exigent de considérer une distance limitée entre le bâti, afin de ne pas relier entre eux des noyaux d'habitat par l'intermédiaire d'un habitat dispersé ou en ruban. D'où le choix de la distance maximale de $100 \mathrm{~m}$.

Pour une bonne organisation de la "grande ville», il est certainement utile de considérer les agglomérations. Bien que le bâti de la banlieue ne forme pas un tout avec l'agglomération, la population entretient avec elle des liens étroits en matière de migrations, de déplacements pour le travail et partiellement même pour les études dans l'enseignement secondaire. Des études (Van Hecke, 1998) ont démontré qu'il y avait aussi des liens étroits entre la ville centrale et sa périphérie pour le commerce de détail et les services.

51 On peut conclure de l'analyse que la stabilité territoriale de la région urbaine est assez grande à travers le temps et forme de ce fait un zonage idéal pour la mise en place d'initiatives pour la gestion collective de certaines problématiques. Bien sûr, d'autres méthodes aboutissent à d'autres délimitations. La «méthode de Louvain» donne un résultat optimisé d'une partition du territoire en "communautés", tandis que la méthode Anabel développée par l'INSEE met plus l'accent sur des structures polarisées (Adam et al., 2018) et mène à des résultats plus ressemblants à ceux obtenus par la méthode suivie ici. 
Figure 1. Les régions urbaines belges (2017).

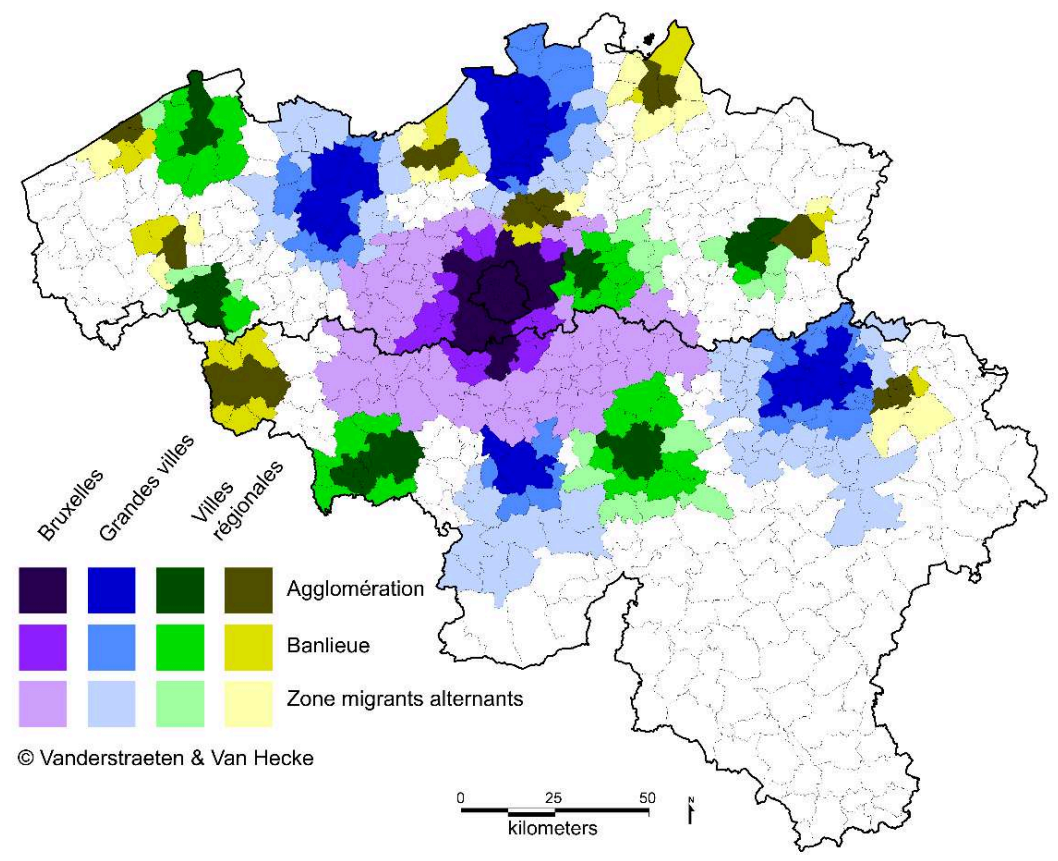

L. Vanderstraeten, E. Van Hecke

Tableau 1. Composition des régions urbaines et des complexes résidentiels urbains et évolution 2001-2017.

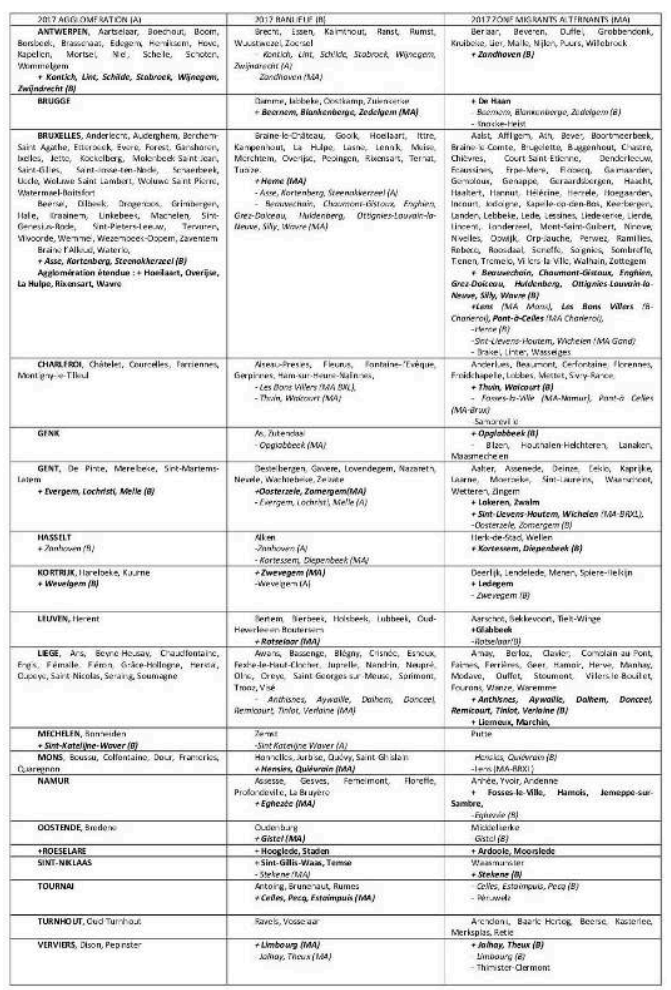


Tableau 2. Population des régions urbaines au 1.1.2017.

\begin{tabular}{|c|c|c|c|c|c|c|}
\hline $\begin{array}{l}\text { POPULATION } \\
2017 \\
\times 1000\end{array}$ & Ville & $\begin{array}{c}\text { Reste de } \mathrm{I}^{\prime} \\
\text { agglomération }\end{array}$ & Agglomération & Banlieue & Région urbaine & $\begin{array}{l}\text { Zone des migrants } \\
\text { alternants }\end{array}$ \\
\hline Bruxelles & 1192 & 497 & 1689 & 214 & 1903 & 1011 \\
\hline Anvers & 520 & 349 & 869 & 142 & 1012 & 234 \\
\hline Gand & 259 & 112 & 371 & 106 & 477 & 229 \\
\hline Bruges & 118 & & 118 & 109 & 228 & 13 \\
\hline Louvain & 100 & 21 & 122 & 80 & 202 & 52 \\
\hline Malines & 86 & 36 & 121 & 23 & 144 & 17 \\
\hline Hasselt & 77 & 21 & 98 & 12 & 110 & 48 \\
\hline Saint-Nicolas & 76 & & 76 & 48 & 124 & 29 \\
\hline Courtrai & 76 & 72 & 148 & 25 & 173 & 62 \\
\hline Ostende & 71 & 18 & 89 & 21 & 110 & 19 \\
\hline Genk & 66 & & 66 & 15 & 81 & 10 \\
\hline Roulers & 62 & & 62 & 21 & 83 & 20 \\
\hline Turnhout & 43 & 13 & 57 & 26 & 83 & 72 \\
\hline Flandre & 1555 & 643 & 2197 & 629 & 2827 & 805 \\
\hline Liège & 198 & 302 & 500 & 131 & 631 & 162 \\
\hline Charleroi & 201 & 89 & 290 & 77 & 368 & 96 \\
\hline Namur & 111 & & 111 & 67 & 178 & 80 \\
\hline Mons & 95 & 98 & 193 & 61 & 254 & \\
\hline Tournai & 69 & & 69 & 43 & 112 & \\
\hline Verviers & 55 & 25 & 80 & 6 & 86 & 21 \\
\hline Wallonie & 730 & 514 & 1244 & 385 & 1629 & 359 \\
\hline Belgique & 3476 & 1654 & 5130 & 1229 & 6359 & 2175 \\
\hline $\begin{array}{l}\text { \%de la population } \\
\text { totale de la Belgique }\end{array}$ & $30,7 \%$ & 14,6 & 45,3 & 10,8 & 56,2 & 19,0 \\
\hline
\end{tabular}

Source : Statbel

Tableau 3. Superficie des régions urbaines.

\begin{tabular}{|l|c|c|c|c|c|c|}
\hline $\begin{array}{l}\text { Superficie } \\
\mathbf{k m}^{2}\end{array}$ & Ville & $\begin{array}{c}\text { Reste de } \\
\text { Yagglomération }\end{array}$ & Agglomération & Banlieue & Région urbaine & $\begin{array}{c}\text { Zone des migrants } \\
\text { alternants }\end{array}$ \\
\hline Bruxelles & $\mathbf{1 6 1}$ & $\mathbf{5 2 0}$ & $\mathbf{6 8 1}$ & $\mathbf{5 1 6}$ & $\mathbf{1 1 9 7}$ & $\mathbf{2 9 1 0}$ \\
\hline Anvers & 204 & 314 & 518 & 389 & 907 & 501 \\
\hline Gand & 156 & 220 & 376 & 295 & 671 & 686 \\
\hline Villes régionales & 768 & 267 & 1035 & 1227 & 2262 & 1005 \\
\hline Flandre & $\mathbf{1 1 2 8}$ & $\mathbf{8 0 1}$ & $\mathbf{1 9 2 9}$ & $\mathbf{1 9 1 1}$ & $\mathbf{3 8 4 0}$ & $\mathbf{2 1 9 2}$ \\
\hline Liège & 69 & 298 & 367 & 448 & 815 & 1187 \\
\hline Charleroi & 102 & 97 & 199 & 203 & 402 & 834 \\
\hline Villes régionales & 570 & 143 & 713 & 996 & 1709 & 586 \\
\hline Wallonie & $\mathbf{7 4 1}$ & $\mathbf{5 3 8}$ & $\mathbf{1 2 7 9}$ & $\mathbf{1 6 4 7}$ & $\mathbf{2 9 2 6}$ & $\mathbf{2 6 0 7}$ \\
\hline Belgique & $\mathbf{2 0 3 0}$ & $\mathbf{1 8 5 9}$ & $\mathbf{3 8 8 9}$ & $\mathbf{4 0 7 4}$ & $\mathbf{7 9 6 3}$ & $\mathbf{7 7 0 9}$ \\
\hline $\begin{array}{l}\text { \% superficie } \\
\text { Belgique }\end{array}$ & $\mathbf{6 , 6 \%}$ & $\mathbf{6 , 1}$ & $\mathbf{1 2 , 7}$ & $\mathbf{1 3 , 3}$ & $\mathbf{2 6 , 1}$ & $\mathbf{2 5 , 3}$ \\
\hline
\end{tabular}

Source : Statbel

Tableau 4. Evolution de la superficie des régions urbaines selon les Régions : 2001-2017.

\begin{tabular}{|l|c|c|c|c|c|}
\hline $\begin{array}{l}\text { Evolution de la superficie } \\
\text { 2017/2001 (2001=100) }\end{array}$ & Agglomération & Banlieue & $\begin{array}{l}\text { Région } \\
\text { urbaine }\end{array}$ & $\begin{array}{c}\text { Zone des } \\
\text { migrants } \\
\text { altemants }\end{array}$ & $\begin{array}{c}\text { Complexe ésidentiel } \\
\text { urbain }\end{array}$ \\
\hline Région Bruxelles-Cap. & 119 & 55 & 79 & 112 & 100 \\
\hline Flandre & 124 & 95 & 108 & 85 & 98 \\
\hline Wallonie & 100 & 81 & 88 & 133 & 105 \\
\hline Belgique & 114 & 82 & 95 & 108 & 101 \\
\hline $\begin{array}{l}\% \text { superficie } \\
\text { Belgique }\end{array} 2016$ & $12,7 \%$ & 13,3 & 26,1 & 25,3 & 51,3 \\
\hline 2001 & $11,2 \%$ & 16,3 & 27,5 & 23,4 & 50,9 \\
\hline
\end{tabular}

Source : Statbel

Tableau 5. Densité de population : habitants par km².

\begin{tabular}{|l|c|c|c|c|c|c|}
\hline $\begin{array}{l}\text { Habitants/ } \\
\mathbf{k m}^{2}\end{array}$ & Ville & Reste de l'agglomération & Agglomération & Banlieue & Région urbaine & $\begin{array}{c}\text { Zone des migrants } \\
\text { alternants }\end{array}$ \\
\hline Bruxelles & $\mathbf{7 3 8 4}$ & $\mathbf{9 5 7}$ & $\mathbf{2 4 8 0}$ & $\mathbf{4 1 5}$ & $\mathbf{1 5 9 0}$ & $\mathbf{3 4 7}$ \\
\hline Anvers & 2545 & 1110 & 1678 & 366 & & 467 \\
\hline Gand & 1659 & 510 & 987 & 360 & & 334 \\
\hline Villes régionales & 1009 & $\mathbf{6 8 0}$ & 924 & 310 & 592 & 233 \\
\hline Flandre & $\mathbf{1 3 7 8}$ & $\mathbf{8 0 2}$ & $\mathbf{1 1 3 9}$ & $\mathbf{3 2 9}$ & $\mathbf{7 3 6}$ & $\mathbf{3 6 7}$ \\
\hline Liège & 2852 & 1015 & 1362 & 293 & & 137 \\
\hline Charleroi & 1972 & 921 & 1459 & 382 & & 115 \\
\hline Villes régionales & 579 & $\mathbf{9 6 0}$ & 635 & 178 & 369 & 172 \\
\hline Wallonie & $\mathbf{9 8 5}$ & $\mathbf{9 5 6}$ & $\mathbf{9 7 3}$ & $\mathbf{2 3 4}$ & $\mathbf{5 5 7}$ & $\mathbf{1 3 8}$ \\
\hline Belgique & $\mathbf{1 7 1 2}$ & $\mathbf{8 9 0}$ & $\mathbf{1 3 1 9}$ & $\mathbf{2 9 7}$ & $\mathbf{7 9 9}$ & $\mathbf{2 8 2}$ \\
\hline
\end{tabular}

Source : Statbel 
Tableau 6. Emploi 2014.

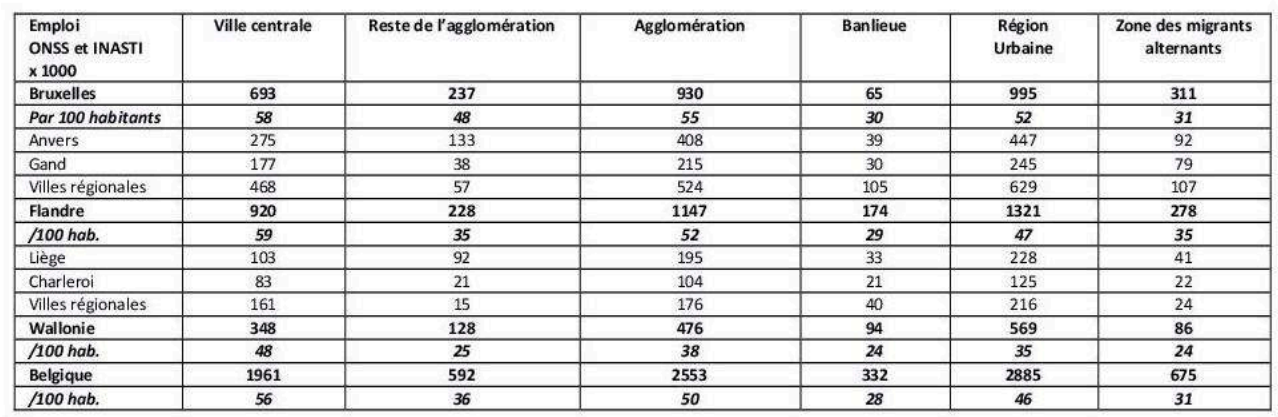

Total Belgique 4502359 dont : hors complexes urbains : 943052 ou 21 \% du total et 33 emplois par 100 habitants).

Source : Salariés : ONSS; indépendants : INASTI

Tableau 7. Revenu moyen par habitant, 2015.

\begin{tabular}{|c|c|c|c|c|c|c|}
\hline $\begin{array}{c}\text { Revenu } \\
\text { moyen 2015 } \\
\text { EUR/hab. }\end{array}$ & Ville & $\begin{array}{c}\text { Reste de } \\
\text { l'agglomération }\end{array}$ & Agglomération & Banlieue & $\begin{array}{c}\text { Région } \\
\text { urbaine }\end{array}$ & $\begin{array}{c}\text { Zone des } \\
\text { migrants alternants }\end{array}$ \\
\hline Bruxelles & $\mathbf{1 3 8 3 1}$ & $\mathbf{2 0 1 7 9}$ & $\mathbf{1 5 6 9 2}$ & $\mathbf{2 1 3 5 9}$ & $\mathbf{1 6 3 3 0}$ & $\mathbf{1 9 5 7 3}$ \\
\hline Anvers & 15688 & 21053 & 17844 & 19900 & 18133 & 19574 \\
\hline Gand & 17923 & 21758 & 19081 & 20369 & 19368 & 19254 \\
\hline Bruges & 19754 & & 19754 & 19190 & 19468 & 20536 \\
\hline Louvain & 20401 & 23722 & 20986 & 23143 & 21843 & 20132 \\
\hline Malines & 18615 & 21921 & 19589 & 23058 & 20149 & 19591 \\
\hline Hasselt & 20014 & 18848 & 19760 & 19274 & 19709 & 19138 \\
\hline Saint-Nicolas & 17688 & & 17688 & 19013 & 18027 & 19740 \\
\hline Courtrai & 18543 & 18062 & 18306 & 19046 & 18413 & 17279 \\
\hline Ostende & 17808 & 18724 & 17988 & 18084 & 18007 & 18176 \\
\hline Genk & 16160 & & 16160 & 18943 & 16689 & 17938 \\
\hline Roulers & 18198 & & 18198 & 17508 & 18020 & 17463 \\
\hline Turnhout & 17459 & 20371 & 18147 & 19161 & 18465 & 18632 \\
\hline $\begin{array}{c}\text { Région } \\
\text { flamande }\end{array}$ & $\mathbf{1 7 5 5 0}$ & $\mathbf{2 0 8 2 4}$ & $\mathbf{1 8 5 0 8}$ & $\mathbf{2 0 0 7 5}$ & $\mathbf{1 8 8 5 8}$ & $\mathbf{1 9 1 4 5}$ \\
\hline Liège & 14533 & 15621 & 15190 & 19212 & 16033 & 18185 \\
\hline Charleroi & 13057 & 14374 & 13460 & 17176 & 14241 & 16873 \\
\hline Namur & 17639 & & 17639 & 19432 & 18315 & 16941 \\
\hline Mons & 15678 & 13993 & 14822 & 16988 & 15339 & \\
\hline Tournai & 16794 & & 16794 & 16314 & 16612 & 189010 \\
\hline Verviers & 13948 & 13666 & 13861 & 16015 & 14010 & 17604 \\
\hline $\begin{array}{c}\text { Région } \\
\text { wallonne }\end{array}$ & $\mathbf{1 4 9 1 5}$ & $\mathbf{1 4 9 9 9}$ & $\mathbf{1 4 9 5 0}$ & $\mathbf{1 8 1 1 9}$ & $\mathbf{1 7 0 8 5}$ & $\mathbf{1 7 6}$ \\
\hline Belgique & $\mathbf{1 5 7 1 8}$ & $\mathbf{1 8 8 1 4}$ & $\mathbf{1 6 7 1 6}$ & $\mathbf{1 9 7 1 0}$ & $\mathbf{1 7 2 8 3}$ & $\mathbf{1 9 0 8 9}$ \\
\hline
\end{tabular}

Source : Statbel 
Tableau 8. Évolution de la population à l'intérieur des agglomérations '2017'.

\begin{tabular}{|c|c|c|c|c|c|c|c|}
\hline $\begin{array}{c}\text { Population et } \\
\text { accroissement } \times 1000\end{array}$ & & 1970 & 2001 & 2017 & $\begin{array}{c}\text { Evolution } \\
\text { totale } 1970- \\
2001 \\
\end{array}$ & $\begin{array}{r}\text { Evolution } \\
\text { totale 2001-17 }\end{array}$ & $\begin{array}{l}\text { Evolution } \\
\text { étrangers 2001- } \\
2017 \\
\end{array}$ \\
\hline \multirow[t]{4}{*}{ ANVERS } & Ville & 542 & 446 & 521 & -96 & +75 & +52 \\
\hline & Reste agglom. 1970 & 100 & 110 & 113 & +10 & +3 & +4 \\
\hline & $\begin{array}{l}\text { Accroissement agglom. } \\
\text { de } 1981 \text { a } 2017\end{array}$ & 175 & 220 & 235 & +45 & +15 & +6 \\
\hline & Agglom. 2017 & 817 & 7776 & 869 & -41 & +93 & +63 \\
\hline \multirow[t]{4}{*}{ GAND } & Ville & 256 & 225 & 259 & -31 & +34 & +21 \\
\hline & Reste agglom. 1970 & 18 & 22 & 25 & +4 & +3 & +1 \\
\hline & Agglom. $1981 \Rightarrow 2017$ & 63 & 79 & 87 & +16 & +8 & +1 \\
\hline & Agglom. 2017 & 337 & 326 & 371 & -11 & +45 & +23 \\
\hline \multirow[t]{4}{*}{ LIEGE } & Ville & 243 & 184 & 198 & -59 & +13 & +8 \\
\hline & Reste agglom. 1970 & 256 & 249 & 260 & -7 & +11 & -5 \\
\hline & Agglom. $1981 \Rightarrow 2017$ & 30 & 38 & 42 & +8 & +3 & - \\
\hline & Agglom. 2017 & 529 & 472 & 500 & -57 & +28 & +3 \\
\hline \multirow[t]{3}{*}{ CHARLEROI } & Ville & 237 & 200 & 201 & -37 & +1 & - \\
\hline & Reste agglom. 1970 & 94 & 87 & 89 & -7 & +2 & -2 \\
\hline & Agglom. 2017 & 331 & 287 & 290 & -44 & +3 & -2 \\
\hline \multirow[t]{4}{*}{ BRUXELLES } & Région Bxl.-Cap. & 1075 & 964 & 1192 & -111 & +227 & +151 \\
\hline & Reste agglom. 1970 & 259 & 317 & 357 & +58 & +39 & +21 \\
\hline & Agglom. $1981 \Rightarrow 2017$ & 102 & 123 & 140 & +21 & +18 & +8 \\
\hline & Agglom. 2017 & 1437 & 1405 & 1689 & -32 & +284 & +180 \\
\hline \multirow[t]{4}{*}{$\begin{array}{l}\begin{array}{r}\text { Villes } \\
\text { Flandre }\end{array} \\
\end{array}$} & Villes & 704 & 715 & 775 & +11 & +60 & +36 \\
\hline & Reste agglom. 1970 & 36 & 39 & 41 & +3 & +2 & +1 \\
\hline & Agglom. $1981 \Rightarrow 2017$ & 131 & 167 & 180 & +36 & +14 & +4 \\
\hline & Agglom. 2017 & 871 & 922 & 997 & +50 & +75 & +40 \\
\hline \multirow[t]{4}{*}{$\begin{array}{l}\text { Villes régionales } \\
\text { Wallonie }\end{array}$} & Villes & 317 & 316 & 331 & -1 & +14 & +11 \\
\hline & Reste agglom. 1970 & 105 & 94 & 97 & -11 & +2 & -1 \\
\hline & Agglom. $1981 \Rightarrow 2017$ & 27 & 26 & 27 & -1 & - & - \\
\hline & Agglom. 2017 & 450 & 437 & 454 & -13 & +17 & +10 \\
\hline
\end{tabular}

Sources : INS, Statbel

Tableau 9. Évolution 1970-2001 de la population des régions urbaines (délimitation 2017).

\begin{tabular}{|c|c|c|c|c|c|}
\hline $\begin{array}{c}\text { Population } \\
2001 / 1970=100\end{array}$ & Ville centrale & $\begin{array}{c}\text { Reste de } \\
\text { Pagglomération }\end{array}$ & Banlieue & Région urbaine & $\begin{array}{c}\text { Zone des migrants } \\
\text { alternants }\end{array}$ \\
\hline Bruxelles & $\mathbf{9 0}$ & $\mathbf{1 2 2}$ & $\mathbf{1 3 2}$ & $\mathbf{1 0 1}$ & $\mathbf{1 1 6}$ \\
\hline Anvers & 81 & 123 & 154 & 100,5 & 114 \\
\hline Gand & $\mathbf{8 8}$ & 125 & 117 & 101 & 108 \\
\hline $\begin{array}{c}\text { Villes régionales } \\
\text { Flandre }\end{array}$ & 102 & 126 & 127 & 111 & 120 \\
\hline Flandre & $\mathbf{9 2}$ & $\mathbf{1 2 4}$ & $\mathbf{1 3 0}$ & $\mathbf{1 0 5}$ & $\mathbf{1 1 5}$ \\
\hline Liège & $\mathbf{7 6}$ & 100 & 125 & 95 & 121 \\
\hline Charleroi & 85 & 92 & 106 & 90 & 114 \\
\hline $\begin{array}{c}\text { Villes régionales } \\
\text { Wallonie }\end{array}$ & 100 & 91 & 111 & 100,5 & 122 \\
\hline Wallonie & $\mathbf{8 8}$ & $\mathbf{9 6}$ & $\mathbf{1 0 7}$ & $\mathbf{9 8}$ & $\mathbf{1 1 9}$ \\
\hline
\end{tabular}

Sources: INS, Statbel

Tableau 10. Évolution 2001-2017 de la population des régions urbaines (délimitation 2017).

\begin{tabular}{|c|c|c|c|c|c|}
\hline $\begin{array}{c}\text { Population } \\
2017 / 2001=100\end{array}$ & Ville centrale & Reste agglomération & Banlieue & Région urbaine & $\begin{array}{c}\text { Zone migrants } \\
\text { alternants }\end{array}$ \\
\hline Bruxelles & $\mathbf{1 2 4}$ & $\mathbf{1 1 3}$ & $\mathbf{1 0 8}$ & $\mathbf{1 1 9}$ & $\mathbf{1 1 2}$ \\
\hline Anvers & 117 & 105 & 110 & 112 & 110 \\
\hline Gand & 115 & 111 & 105 & 112 & 109 \\
\hline $\begin{array}{l}\text { Villes régionales } \\
\text { Flandre }\end{array}$ & 108 & 107 & 108 & 108 & 108 \\
\hline Flandre & $\mathbf{1 1 2}$ & $\mathbf{1 0 7}$ & $\mathbf{1 0 8}$ & $\mathbf{1 1 0}$ & $\mathbf{1 0 9}$ \\
\hline Liège & 107 & 105 & 108 & 106 & 112 \\
\hline Charleroi & 100 & 103 & 102 & 102 & 107 \\
\hline $\begin{array}{l}\text { Villes régionales } \\
\text { Wallonie }\end{array}$ & 105 & 103 & 110 & 106 & 112 \\
\hline Wallonie & $\mathbf{1 0 4}$ & $\mathbf{1 0 4}$ & $\mathbf{1 0 8}$ & $\mathbf{1 0 5}$ & $\mathbf{1 1 1}$ \\
\hline
\end{tabular}

Sources : INS, Statbel 


\section{BIBLIOGRAPHIE}

ADAM A., CHARLIER J., DEBUISSON M., DUPREZ J.-P., REGINSTER I. \& THOMAS I. (2018), « Bassins résidentiels en Belgique : deux methodes, une réalité ?», L'espace géographique, 1, pp. 35-50.

CABUS P., VANDERHALLEN D. \& VAN HECKE E. (2014), Verstedelijking en ruimtelijke ordening in Vlaanderen : een update, LUCON \& Afdeling Geografie, KU. Leuven.

CHARLIER J., DEBUISSON M., DUPREZ J.-P. \& REGINSTER I. (2016), « Mouvements résidentiels en Wallonie (1994-2014) : analyse des migrations intercommunales et construction de bassins résidentiels », Working Paper de l'IWEPS, 21.

GRIPPA T. et al. (s.d.), Dynamique des quartiers en difficulté dans les régions urbaines belges, SPP IS, Politique des grandes villes, Igeat-ULB et EES-KULeuven, $87 \mathrm{p}$.

LEEMANS S., PATTYN M., ROUSSEAU S. \& VAN DER HAEGEN H. (1987), « Les régions urbaines belges en 1981 », Études Statistiques, 89, Institut National de Statistique, Bruxelles.

LUYTEN S., VAN HECKE E. (2007), «Les régions urbaines belges 2001 », Statistics Belgium Working Paper, 14, Bruxelles, Institut national de Statistique.

MÉRENNE-SCHOUMAKER B., VAN DER HAEGEN H. \& VAN HECKE E. (1998), « Urbanisation », Monographie 11A, Recensement général de la population et des logements au 1er mars 1991, Institut national de Statistique, Bruxelles.

STATISTICS BELGIUM (2016), « Belgique : une mega-agglomération et beaucoup de plus petites », Nouvelles, 26 janvier.

THOMAS I., VANNESTE D. \& QUERRIAU X. (2011), Habitat, Atlas de Belgique, tome 2, Academia Press, Gent, $78 \mathrm{p}$.

THOMAS I., COTTEELS C., JONES J. \& PEETERS D. (2012), « Revisiting the extension of the Brussels urban agglomeration: new methods, new data, ... new results?», Belgeo, 1-2.

VAN DER HAEGEN H., PATTYN M. (1979), « Les régions urbaines belges », Bulletin de Statistique, 3, Bruxelles, Institut National de Statistique, pp. 235-249.

VAN DER HAEGEN H., VAN HECKE E. \& JUCHTMANS G. (1996), « Les Régions urbaines belges 1991 », Etudes Statistiques, 104, Bruxelles, Institut National de Statistique, pp. 3-42.

VAN HECKE E. (1998), « Actualisation de la hiérarchie urbaine belge », Bulletin du Crédit communal, 3, pp. 45-76.

VAN HECKE E., VANDERSTARETEN L. (2016), « Stadsgewesten in beweging », in DE GRANDE H., VANDENHEEDE H. (eds.), Les défis démographiques de Patrick, Liber Amicorum Prof.Patrick Deboosere, Bruxelles, pp. 76-86.

VERHETSEL A., BECKERS J. \& DE MEYERE M. (2018), « Assessing Daily Urban Systems: A Heterogeneous Commuting Network Approach », Networks and Spatial Economics, Springer, 2018 DOI 10.1007/s11067-018-9425-y.

VIEILLARD-BARON H. (2011), « Banlieue, quartier, ghetto : de l'ambiguité des définitions aux représentations ", Revue de psychosociologie, 2, ERES, 304 p.

VIEILLARD-BARON H. (s.d.), « Banlieue », http://www.hypergeo.eu. 


\section{NOTES}

1. Note technique : secteur statistique, noyau d'habitat, agglomération, localité.

Les secteurs statistiques ont été introduits suite à l'initiative des géographes dans le but de mieux cerner les caractéristiques démographiques et socio-économiques à une échelle infracommunale. Ils ont été définis et utilisés pour la première fois lors du dépouillement et de la publication des résultats du recensement de la population de 1970. Ainsi la Belgique des 589 communes (1977) est subdivisée en environ vingt mille secteurs statistiques. Ceci devait largement compenser l'effet des fusions de communes (2 359 communes avant les fusions) et fournir donc des informations à un micro-niveau.

Lors de la délimitation des secteurs statistiques, la distinction a été faite entre «habitat aggloméré » et « habitat dispersé ». L'institut National de Statistique a sur cette base délimité les «noyaux d'habitat». Un noyau d'habitat est donc un ensemble de secteurs statistiques avec habitat aggloméré, contigu. Les limites de ceux-ci sont indépendants des limites communales. Ce sont des entités morphologiques. Les agglomérations des régions urbaines de 2001 ont été définies sur la base de l'habitat continu $(<200 \mathrm{~m})$, mais avec une restriction, notamment que des concentrations d'habitat soient au moins reliées entre elles par 2 routes dans le but d'éliminer l'effet néfaste de l'habitat en ruban. Il faut toutefois souligner que tout le bâti et même l'urbain est pris en compte: c'est-à-dire que les noyaux d'habitat englobent également des espaces industriels, d'infrastructures routières, d'infrastructures sportives et même de parcs urbains. Ce sont donc des «noyaux urbains ». Les délimitations des noyaux et donc des agglomérations sont ajustées aux limites des secteurs statistiques et ce pour des raisons évidentes d'utilisation des données statistiques disponibles à ce niveau.

Les « agglomérations » ou « localités » définies par Statistics Belgium dans le cadre du règlement européen suivent une tout autre méthode. En contraste avec les noyaux d'habitat définis sur la base de documents de terrain - cartes et photographies aériennes -, les entités morphologiques sont définies automatiquement sur la base d'un algorithme de maximum 200 mètres entre deux habitations. Les entités morphologiques formées tiennent seulement compte de l'habitat, car l'exercice est basé sur les coordonnées de celui-ci. Les autres éléments urbains ne sont pas pris en compte, ce qui donne des rupture entre noyaux à cause de la présence d'infrastructures de transport ou de zones industrielles, etc. Comme la délimitation ne prend pas ces fonctions en compte, les délimitations d'Anvers ou Gand par exemple sont pour le moins surprenantes, une grande partie des installations portuaires n'apparaissant pas dans les agglomérations calculées seulement sur la base de l'habitat.

Les agglomérations présentées ici sont une actualisation basée en premier lieu sur les «agglomérations » ou « localités » de Statistics Belgium, mais avec un algorithme de $100 \mathrm{~m}$ entre habitations, après quoi ces délimitations sont ajustées aux secteurs statistiques superposés à condition qu'au moins $50 \%$ de la population du secteur statistique fasse partie de l'agglomération. Ensuite, d'éventuelles ruptures causées par l'absence d'habitations à moins de 100 mètres sont analysées : si la scission est causée par la présence d'infrastructures, de terrains d'activités économiques, sportives ou récréatives, les ruptures ne sont pas prises en compte et les secteurs statistiques habités de plus de 500 habitants $/ \mathrm{km}^{2}$ sont rattachés à l'agglomération urbaine. En utilisant un seuil minimum de densité, on évite de rattacher à l'agglomération morphologique déjà formée des secteurs statistiques avec un habitat relativement dispersé ou de petits noyaux reliés entre eux par un habitat en ruban. De plus, cette méthode de travail est accompagnée de l'étude de cartes récentes pour éviter des erreurs. Toutefois, on ne peut nier qu'il reste une certaine marge d'interprétation, mais celle-ci est jugée plus fiable qu'une délimitation purement automatique et ne tenant compte que de l'habitat. 


\section{RÉSUMÉS}

Les régions urbaines de la Belgique ont été définies pour la première fois sur la base des données du recensement de 1970 et ensuite sur celles de 1981, 1991, 2001. La révision présentée est basée sur diverses données de 2014 à 2017. En 2017, 19 grandes villes ou villes régionales ont développé une région urbaine composée d'une agglomération, entité d'habitat continu, et d'une banlieue, zone à caractère résidentiel dont la population est fortement orientée vers l'agglomération. Suite à la dynamique urbaine, les agglomérations ont grandi et sont pour les grandes villes composées de plusieurs communes, dont certaines étaient auparavant des communes de banlieue. Les 19 communes-villes sont le cœur de 19 agglomérations comptant 115 communes et entourées de 99 communes de banlieue. Tout comme en 2001, $56 \%$ de la population de la Belgique habite une région urbaine. $64 \%$ de l'emploi est concentré dans ces régions urbaines. Après une longue période d'exode de la population au départ des villes centrales de 1970 à la fin des années 1990, on assiste à une nette augmentation de la population des villes-centres entre 2001 et 2017. Ce retournement de situation est dû à l'immigration étrangère mais non exclusivement. Finalement une importante évolution se marque dans la périphérie: pendant la période 1970-2001, l'accroissement de la population de la banlieue était deux fois plus important que celui dans la zone des migrants alternants (navetteurs). Après 2000, le taux d'accroissement est de même niveau, ce qui signifie une tendance néfaste pour l'ampleur des déplacements.

The Belgian urban regions were defined for the first time in the early 1970s. Later on they were updated based on the censuses of 1981, 1991 and 2001. The current update is based on administrative data varying between 2014 and 2017. In 2017, 19 cities in Belgium developed an urban region with on the one hand an agglomeration (a zone of uninterrupted built environment) and on the other hand an urban fringe (a zone of more dispersed residential settlements but with a strong focus of the population on the agglomeration). The Belgian urban regions concentrate $56 \%$ of the population and $64 \%$ of the employment. After a long period of a netto exodus from the centres of the urban regions in Belgium, one can see a growth of the population of the centers between 2001 and 2017. Immigration from abroad plays an important role in this netto growth, but more issues are at stake. A final important reversal takes place in the periphery of the urban regions. Between 1970 and 2001, the growth of the populations in the urban fringes was twice as high compared to the growth in the commuter zones. Between 2001 and 2017 , an equal growth in both zones took place, meaning that the sprawl of the population did not stop and takes as much place at a longer distance from the centres of the urban regions then at a shorter distance.

\section{INDEX}

Keywords : urban region, city, agglomeration, Belgium

Mots-clés : région urbaine, ville, agglomération, banlieue, Belgique

\section{AUTEURS}

\section{LIEVE VANDERSTRAETEN}

Cosmopolis Centre for Urban Research, Vakgroep geografie, Vrije Universiteit Brussel, Lieve.Vanderstraeten@vub.be 


\section{ETIENNE VAN HECKE}

Afdeling Geografie en Toerisme, KULeuven, etienne.vanhecke@kuleuven.be 